\title{
Circulating ESR1 mutations at the end of aromatase inhibitor adjuvant treatment and after relapse in breast cancer patients
}

Violette Allouchery ${ }^{1 \dagger}$, Ludivine Beaussire ${ }^{2 \dagger}$, Anne Perdrix ${ }^{2,3}$, David Sefrioui ${ }^{2,4}$, Laetitia Augusto ${ }^{1}$, Cécile Guillemet ${ }^{1}$, Nasrin Sarafan-Vasseur ${ }^{2}$, Frédéric Di Fiore ${ }^{1,2,4}$ and Florian Clatot $^{1,2^{*+}}$ (I)

\begin{abstract}
Background: Detection of circulating ESR1 mutations is associated with acquired resistance to aromatase inhibitor (Al) in metastatic breast cancer. Until now, the presence of circulating ESR1 mutations at the end of adjuvant treatment by $\mathrm{Al}$ in early breast cancer had never been clearly established. In this context, the aim of the present study was to evaluate the circulating ESR1 mutation frequency at the end of adjuvant treatment and after relapse.

Methods: This monocentric retrospective study was based on available stored plasmas and included all early breast cancer patients who completed at least 2 years of Al adjuvant treatment and experienced a documented relapse after the end of their treatment. Circulating ESR1 mutations (D538G, Y537S/N/C) were assessed by droplet digital PCR in plasma samples taken at the end of adjuvant treatment, at time of relapse and at time of progression under first line metastatic treatment.

Results: A total of 42 patients were included, with a median adjuvant Al exposure of 60 months (range 41-85). No circulating ESR1 mutation was detectable at the end of Al adjuvant therapy. At first relapse, 5.3\% of the patients (2/38) had a detectable circulating ESR1 mutation. At time of progression on first-line metastatic treatment, 33\% of the patients (7/21) under Al had a detectable circulating ESR1 mutation compared to none of the patients under chemotherapy (0/10). The two patients with a detectable ESR1 mutation at relapse were treated by Al and had an increase of their variant allele fraction at time of progression on first-line metastatic treatment.

Conclusions: Circulating ESR1 mutation detection at the end of Al-based adjuvant treatment is not clinically useful. Circulating ESR1 mutation could be assessed as soon as first relapse to guide interventional studies.
\end{abstract}

Keywords: Early breast cancer, ESR1 mutation, Circulating tumor DNA, Aromatase inhibitor resistance, Digital PCR

\section{Introduction}

Aromatase inhibitors (AI) are a key treatment in postmenopausal hormone receptor positive $(\mathrm{HR}+)$ breast cancer (BC). Estrogen receptor (ESR1) mutation emergence has been recently highlighted as a frequent mechanism of acquired AI resistance in the metastatic setting, as well as a prognostic marker of poor outcome [1-3].

\footnotetext{
* Correspondence: florian.clatot@chb.unicancer.fr

${ }^{\dagger}$ Equal contributors

${ }^{1}$ Department of Medical Oncology, Centre Henri Becquerel, 1 rue d'Amiens, 76038 Rouen cedex 1, France

${ }^{2}$ Normandie Univ, UNIROUEN, Inserm U1245, IRON group, Rouen University Hospital, Normandy Centre for Genomic and Personalized Medicine, Rouen, France

Full list of author information is available at the end of the article
}

ESR1 mutations are characterized by a frequency of no more than $2 \%$ in primary tumour [4] compared to $30 \%$ in metastatic tissues among AI-resistant patients [1, 3]. Furthermore, ESR1 mutations located in four hot-spots (Y537N/S/C, D538G) count for $74 \%$ of all described mutations [5], and can be successfully detected in circulating tumour DNA (ctDNA) [6, 7].

Besides its use in the metastatic setting, AI are widely administered as adjuvant treatment of $\mathrm{HR}+$ early $\mathrm{BC}$ $(E B C)$ with a usual exposure of 5 years [8]. Despite this long time of exposure, data concerning circulating ESR1 mutation emergence under adjuvant AI therapy are lacking. It has been reported that circulating ESR1 mutations at the time of metastatic diagnosis in patients previously exposed

(C) The Author(s). 2018 Open Access This article is distributed under the terms of the Creative Commons Attribution 4.0 International License (http://creativecommons.org/licenses/by/4.0/), which permits unrestricted use, distribution, and 
to $\mathrm{AI}$ in the adjuvant setting may be detected at rates ranging from 2.6 [6] to $11.3 \%$ [1]. Until now, circulating ESR1 mutation emergence between adjuvant treatment ending, relapse and AI re-exposure remains not clearly established.

In this context, the aim of the present study was to evaluate the ESR1 circulating mutation frequency at the end of AI adjuvant treatment in EBC patients with a subsequent local or metastatic relapse.

\section{Patients and methods}

\section{Patients}

We retrospectively screened $\mathrm{HR}+\mathrm{EBC}$ patients treated from 2008 to 2014 by adjuvant AI for at least 2 years and who subsequently experienced a documented localized or metastatic relapse. Only patients with available blood sample collection during the last 6 months of $\mathrm{AI}$ in the adjuvant setting were included in the analysis.

\section{Droplet digital PCR analysis}

Analyses for circulating ESR1 mutation detection were performed blind to clinical data and using the same methods as we previously reported [2]. Complete methods are provided as supplementary data (available online). Briefly, after a first step of total plasma DNA extraction and quantification, a droplet-based ddPCR platform (Qx200 ${ }^{\circ}$ ddPCR System, Bio-rad Laboratories, Hercules, CA, USA) was used for detection of four ESR1 mutations: Y537N, Y537S, Y537C and D538G.

\section{Statistical analysis}

The primary objective was to determine the rate of circulating ESR1 mutation detection at the end of AI-based adjuvant treatment. In cases with available samples, we also assessed the rate of circulating ESR1 mutation at time of relapse and time of progression to the first-line treatment used in metastatic setting.

\section{Results}

\section{Patients characteristics}

The main characteristics of the 42 patients included are summarized in Table 1. A flow chart of the study is provided in Fig. 1. After relapse, 24 (63\%) patients received AI as first-line metastatic treatment. For the remaining 14 patients, six were treated by non-AI endocrine therapy, four by chemotherapy, two by chemotherapy + anti-HER2 therapy, one by palliative care, and finally the patient with local recurrence benefited from surgery followed by adjuvant chemotherapy.

\section{Circulating ESR1 mutational status at the end of the adjuvant phase and at progression during first-line Al in metastatic phase}

None of the 42 patients included had a circulating ESR1 mutation detectable at the end of AI adjuvant therapy.
Table 1 Characteristics

\begin{tabular}{|c|c|c|}
\hline Characteristics & $\mathrm{N}(\%)$ & \\
\hline $\begin{array}{l}\text { Median age at diagnosis: } \\
\text { years (min-max) }\end{array}$ & $60.7(47-78)$ & \\
\hline \multirow[t]{2}{*}{ Lymph node status } & Positive & $32(76 \%)$ \\
\hline & Negative & $10(24 \%)$ \\
\hline \multirow[t]{3}{*}{ Her2 status } & Positive & $3(7 \%)$ \\
\hline & Negative & $25(60 \%)$ \\
\hline & Unknown & $14(33 \%)$ \\
\hline HR status & Positive & $42(100 \%)$ \\
\hline \multirow[t]{2}{*}{ Neoadjuvant chemotherapy } & Yes & $4(9.5 \%)$ \\
\hline & No & $38(90.5 \%)$ \\
\hline \multirow[t]{2}{*}{ Adjuvant chemotherapy } & Yes & $33(78.6 \%)$ \\
\hline & No & $9(21.4 \%)$ \\
\hline Adjuvant Al treatment & Yes & $42(100 \%)$ \\
\hline \multirow{2}{*}{$\begin{array}{l}\text { Tamoxifen before Al during } \\
\text { adjuvant treatment }\end{array}$} & Yes & $9(21.4 \%)$ \\
\hline & No & $33(78.6 \%)$ \\
\hline $\begin{array}{l}\text { Median duration of Al adjuvant } \\
\text { treatment: months (min-max) }\end{array}$ & $60.0(41-85)$ & \\
\hline $\begin{array}{l}\text { Median delay between end of } \\
\text { adjuvant treatment and relapse: } \\
\text { months (min-max) }\end{array}$ & $25(2-71)$ & \\
\hline \multirow[t]{2}{*}{ Type of relapse } & Local & $1(2.4 \%)$ \\
\hline & Metastatic & $41(97.6 \%)$ \\
\hline $\begin{array}{l}\text { Median duration of follow-up during } \\
\text { metastatic setting: months (min-max) }\end{array}$ & $32.9(1-75)$ & \\
\hline
\end{tabular}

Plasma samples at relapse were available for 38 patients (90\%) of the full cohort. Among them, 2 (5.3\%) had a detectable ESR1 circulating mutation (Y537C and D538G) at time of relapse (Fig. 2). Plasma samples at time of progression on first-line metastatic treatment were available for 31 (74\%) patients, including 21 treated with AI (Fig. 1). A circulating ESR1 mutation was detected in 7/ $21(33 \%)$ patients progressing on AI while none of the ten patients progressing on chemotherapy or non-AI endocrine treatment had a detectable circulating ESR1 mutation. Of note, 3/42 patients had an early relapse ( $<6$ months) after the end of adjuvant AI. In none of these three patients was an ESR1 mutation detected at the end of adjuvant AI or at relapse.

Concerning the two patients with a detectable circulating ESR1 mutation at relapse after adjuvant treatment, the first had adjuvant treatment consisting of 3 years of tamoxifen followed by 4 years of AI. Metastatic relapse occurred 4 years after adjuvant treatment completion, with a Y537C mutation detected at $0.7 \%$ variant allele fraction (VAF) in circulating plasma at time of relapse. After 11 months of first-line AI, a documented clinical progression was observed with the same detectable circulating Y537C mutation, which increased significantly to $15.2 \%$ VAF. 

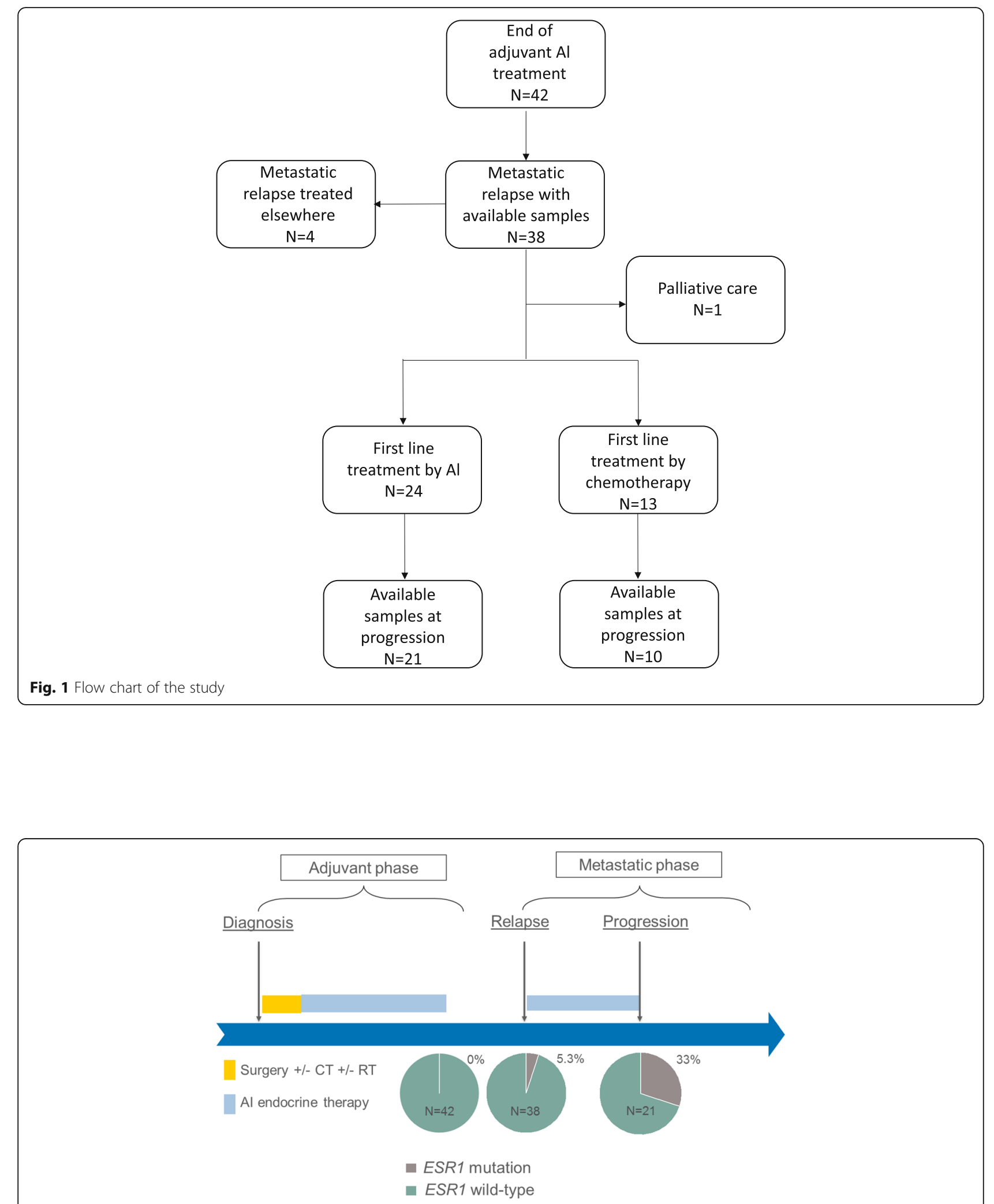

Fig. 2 Circulating ESR1 mutation frequency during HR+ breast cancer history. At the end of adjuvant treatment by Al, circulating ESR1 mutations were not detected among the 42 patients of this cohort. At relapse, 2/38 patients (5.3\%) had a detectable circulating ESR1 mutation. At progression after re-exposure to $\mathrm{Al}$ as first-line metastatic treatment, $7 / 21$ patients (33\%) had a detectable circulating ESR1 mutation. Al aromatase inhibitor, $C T$ chemotherapy, $R T$ radiation therapy 
The second patient with a detectable circulating ESR1 mutation at first relapse received 5 years of AI adjuvant treatment and experienced a metastatic relapse at 20 months of follow-up. The circulating ESR1 D538G mutation was detected at $6.7 \%$ VAF rate at time of relapse and increased to a $14 \%$ VAF at time to progression after 9 months under $\mathrm{AI}$ in the metastatic setting.

\section{Discussion}

To our knowledge, this study is the first addressing the frequency of circulating ESR1 mutation at the end of AI adjuvant treatment in patients treated for an EBC. Our results highlighted that no circulating ESR1 mutation was detectable at the end of AI-based adjuvant treatment in $\mathrm{HR}+\mathrm{EBC}$ patients who also subsequently experienced a relapse during follow-up. In contrast, re-exposure to $\mathrm{AI}$ in the metastatic setting induced circulating ESR1 mutation emergence among $33 \%$ of patients, which was close to the 30-50\% circulating ESR1 mutation frequency usually observed for metastatic patients progressing on AI $[1-3,9]$. Interestingly, we observed that circulating ESR1 mutation was detectable in a small proportion of patients (5.3\%) at metastatic diagnosis, which is also a finding in line with previous data reporting a frequency ranging from 2.6 to $11.3 \%$ in that setting $[1,8]$.

Our study has some inherent limitations due to its retrospective design. First, considering the limited number of patients, the emergence of a circulating ESR1 mutation at the end of the adjuvant phase by AI cannot be formally excluded. Thus, larger prospective studies with pre-defined sample processing are needed to confirm our results. Second, since we focused the analysis on the four main ESR1 mutations, we cannot exclude the presence of rare circulating mutations [10]. In terms of daily practice, our results suggest that screening for a circulating ESR1 mutation detection at the end of adjuvant AI treatment would not be of interest. In contrast, we found that ESR1 mutations at relapse were present in $5.3 \%$ of patients and that their levels increased under AI exposure. Even rare, circulating ESR1 mutation at relapse after an AI exposure limited to the adjuvant setting has already been reported by others [8]. Thus, early treatment change in cases of detected circulating ESR1 mutations should be assessed by clinical trials as soon as metastatic relapse in case of previous AI exposure, and not only after AI treatment in the metastatic setting.

Concerning ESR1 mutation emergence during the metastatic phase, it has to be noted that despite longterm exposure to adjuvant AI (median of 5 years), most of the patients with a detectable ESR1 mutation in the metastatic setting had a detected circulating mutation only after 20 months of AI re-exposure. On the other hand, the observation that two patients harboured ESR1 mutations at relapse confirms the hypothesis that mutational clone emergence was selected by AI therapy during the adjuvant setting, but not detectable at that time given a low tumour burden [8]. Interestingly, these two patients had a comparable delay (20 and 48 months) between end of AI adjuvant treatment and metastatic relapse compared to the other patients of the cohort (median of 25 months).

To conclude, ours results show that detection of circulating ESR1 mutation at the end of AI-based adjuvant treatment in HR+ EBC patients is not clinically useful. Even if technical advances-such as plasmapheresis or use of implanted ultrasensitive devices [11] - may increase the amount of evaluable ctDNA and thus improve sensitivity of mutation detection in the future, ongoing efforts to detect circulating ESR1 mutations should be focused on the metastatic setting.

Funding

Centre Henri Becquerel.

Availability of data and materials

Please contact the corresponding author for additional information on how to obtain the study data.

\section{Authors' contributions}

Design: FDF, FC. Supervision: AP, DS, NSV, FDF, FC. Collection of clinical data: VA, LA, GC, FC. Collection and preparation of biological samples: AP. Experiments: LB, NSV. Data analysis: VA, LB, NSV, FDF, FC. Preparation of the manuscript, table and figures (all originals): VA, FDF, FC. All authors read and approved the final manuscript.

Ethics approval and consent to participate

All patients signed a consent form allowing the conservation and study of their biological samples.

The study was approved by the Institutional Review Board of the Henri Becquerel Center (registering order 1702B).

\section{Competing interests}

The authors declare that they have no competing interests.

\section{Publisher's Note}

Springer Nature remains neutral with regard to jurisdictional claims in published maps and institutional affiliations.

\section{Author details}

${ }^{1}$ Department of Medical Oncology, Centre Henri Becquerel, 1 rue d'Amiens, 76038 Rouen cedex 1, France. ${ }^{2}$ Normandie Univ, UNIROUEN, Inserm U1245, IRON group, Rouen University Hospital, Normandy Centre for Genomic and Personalized Medicine, Rouen, France. ${ }^{3}$ Department of Bio-Pathology, Centre Henri Becquerel, Rouen, France. ${ }^{4}$ Department of Gastroenterology, Rouen University Hospital, Rouen, France.

Published online: 16 May 2018

References

1. Chandarlapaty S, Chen D, He W, Sung P, Samoila A, You D, et al. Prevalence of ESR1 mutations in cell-free DNA and outcomes in metastatic breast cancer: a secondary analysis of the BOLERO-2 clinical trial. JAMA Oncol. 2016;2:1310-5.

2. Clatot F, Perdrix A, Augusto L, Beaussire L, Delacour J, Calbrix C, et al. Kinetics, prognostic and predictive values of ESR1 circulating mutations in metastatic breast cancer patients progressing on aromatase inhibitor. Oncotarget. 2016;7:74448-59.

3. Fribbens C, O'Leary B, Kilburn L, Hrebien S, Garcia-Murillas I, Beaney M, et al. Plasma ESR1 mutations and the treatment of estrogen receptor-positive advanced breast cancer. J Clin Oncol. 2016;34:2961-8. 
4. Takeshita T, Yamamoto Y, Yamamoto-lbusuki M, Inao T, Sueta A, Fujiwara S, et al. Droplet digital polymerase chain reaction assay for screening of ESR1 mutations in 325 breast cancer specimens. Transl Res. 2015;166:540-553.e2.

5. Segal CV, Dowsett M. Estrogen receptor mutations in breast cancer-new focus on an old target. Clin Cancer Res. 2014;20:1724-6.

6. Schiavon G, Hrebien S, Garcia-Murillas I, Cutts RJ, Pearson A, Tarazona N, et al. Analysis of ESR1 mutation in circulating tumor DNA demonstrates evolution during therapy for metastatic breast cancer. Sci Transl Med. 2015; 7:313ra182.

7. Sefrioui D, Perdrix A, Sarafan-Vasseur N, Dolfus C, Dujon A, Picquenot J-M, et al. Short report: Monitoring ESR1 mutations by circulating tumor DNA in aromatase inhibitor resistant metastatic breast cancer. Int J Cancer. 2015; 137:2513-9.

8. Senkus E, Kyriakides S, Ohno S, Penault-Llorca F, Poortmans P, Rutgers E, et al. Primary breast cancer: ESMO Clinical Practice Guidelines for diagnosis, treatment and follow-up. Ann Oncol. 2015;26(Suppl 5):v8-30.

9. Fribbens C, Garcia Murillas I, Beaney M, Hrebien S, O'Leary B, Kilburn L, et al. Tracking evolution of aromatase inhibitor resistance with circulating tumour DNA analysis in metastatic breast cancer. Ann Oncol. 2018;29(1):145-53. https://doi.org/10.1093/annonc/mdx483.

10. Spoerke JM, Gendreau S, Walter K, Qiu J, Wilson TR, Savage H, et al. Heterogeneity and clinical significance of ESR1 mutations in ER-positive metastatic breast cancer patients receiving fulvestrant. Nat Commun. 2016;7:11579.

11. Wan JCM, Massie C, Garcia-Corbacho J, Mouliere F, Brenton JD, Caldas C, et al. Liquid biopsies come of age: towards implementation of circulating tumour DNA. Nat Rev Cancer. 2017;17:223-38. 